\title{
Agroforestry leads to shifts within the gammaproteobacterial microbiome of banana plants cultivated in Central America
}

\author{
Martina Köberl ${ }^{1}$, Miguel Dita ${ }^{2,3}$, Alfonso Martinuz ${ }^{3}$, Charles Staver ${ }^{4}$ and Gabriele Berg ${ }^{1}$ \\ ${ }^{1}$ Institute of Environmental Biotechnology, Graz University of Technology, Graz, Austria \\ ${ }^{2}$ Brazilian Agricultural Research Corporation - Embrapa, Brasília, Brazil \\ ${ }^{3}$ Bioversity International Costa Rica, Turrialba, Costa Rica \\ ${ }^{4}$ Bioversity International France, Montpellier, France
}

Edited by:

Anna Maria Pirttilä, University of Oulu, Finland

\section{Reviewed by:}

David John Studholme, University of Exeter, UK

Nai-Chun Lin, National Taiwan

University, Taiwan

Reindert Nijland, Wageningen

University, Netherlands

\section{*Correspondence:}

Martina Köberl, Institute of

Environmental Biotechnology, Graz

University of Technology,

Petersgasse 12//, 8010 Graz, Austria

e-mail: martina.koeberl@tugraz.at
Bananas (Musa spp.) belong to the most important global food commodities, and their cultivation represents the world's largest monoculture. Although the plant-associated microbiome has substantial influence on plant growth and health, there is a lack of knowledge of the banana microbiome and its influencing factors. We studied the impact of (i) biogeography, and (ii) agroforestry on the banana-associated gammaproteobacterial microbiome analyzing plants grown in smallholder farms in Nicaragua and Costa Rica. Profiles of 16S rRNA genes revealed high abundances of Pseudomonadales, Enterobacteriales, Xanthomonadales, and Legionellales. An extraordinary high diversity of the gammaproteobacterial microbiota was observed within the endophytic microenvironments (endorhiza and pseudostem), which was similar in both countries. Enterobacteria were identified as dominant group of above-ground plant parts (pseudostem and leaves). Neither biogeography nor agroforestry showed a statistically significant impact on the gammaproteobacterial banana microbiome in general. However, indicator species for each microenvironment and country, as well as for plants grown in Coffea intercropping systems with and without agri-silvicultural production of different Fabaceae trees (Inga spp. in Nicaragua and Erythrina poeppigiana in Costa Rica) could be identified. For example, banana plants grown in agroforestry systems were characterized by an increase of potential plant-beneficial bacteria, like Pseudomonas and Stenotrophomonas, and on the other side by a decrease of Erwinia. Hence, this study could show that as a result of legume-based agroforestry the indigenous banana-associated gammaproteobacterial community noticeably shifted.

Keywords: agroforestry, banana-associated Gammaproteobacteria, banana-coffee intercropping, Gros Michel, Musa

\section{INTRODUCTION}

Musa spp., including dessert and cooking bananas, are large perennial monocotyledonous herbs of the order Zingiberales. Their domestication process started about 7000 years ago and involved hybridizations between diverse species and subspecies and the selection of sometimes diploid, but generally triploid seedless, parthenocarpic hybrids, which were thereafter widely dispersed by vegetative propagation (Perrier et al., 2011). The cultigens are landraces and belong to the most important agricultural crops in the tropics and sub-tropics. Worldwide, over 100 million metric tons of fruits are produced annually. Cultivars that enter international commerce are worth $\$ 5$ billion per year, and locally consumed fruits are major staples for 400 million people in Latin America and Africa (FAOSTAT, 2005).

The Musa acuminata cultivar Gros Michel, also known as Big Mike, was the main exported banana variety from the nineteenth century until the late 1950s. However, in response to the susceptibility of this cultivar to the fungal pathogen Fusarium oxysporum f. sp. cubense (Foc) race 1, Gros Michel was widely replaced by the resistant Cavendish variety (Ploetz, 2006; Butler, 2013). In many countries in Central America, such as Costa Rica and Nicaragua, the Gros Michel variety is still grown, mainly by smallholder farmers in banana-coffee intercropping systems, sometimes in combination with agroforestry systems, where a lower disease incidence is reported in comparison to monocultures. Gros Michel fruits are praised for their fabulous flavor and, due to their thicker skin, for a better robustness to bruises in comparison to Cavendish. Agroforestry in general is a collective name for land-use systems in which woody perennials are grown in association with herbaceous plants or livestock, in spatial arrangement, a rotation or both (Lundgren, 1982). These practices are considered as functionally biodiverse, environmentally friendly and sustainable land-use alternatives. It was shown that such systems were able to enhance soil fertility and productivity by improving certain soil physical properties and protective functions, such as nutrient cycling and carbon sequestration 
(Montagnini and Nair, 2004; Seobi et al., 2005; Udawatta et al., 2009). Undeniably, it can be assumed that these environmental benefits are associated with soil microbial activity and soil biological parameters. In addition, the plant-associated microbiome has substantial influence on plant growth, quality, and health (Berg et al., 2014). However, despite the importance of banana for grower's livelihoods on these agroforestry systems in Central America and the hypothesized role of soil and plant microbiome on building healthier environments, knowledge on the microbial diversity of representative productions areas is still scarce.

The objective of this study was to decipher the gammaproteobacterial microbiome of banana plants cultivated in Central America. In order to obtain an almost complete picture of the banana-colonizing Gammaproteobacteria under diverse conditions, different plant parts and microenvironments were investigated: the rhizosphere soil surrounding the roots and represents the interface to the bulk soil, the inner tissue of the roots - the endorhiza, the banana leaves, as well as the pseudostem. The cylindrical succulent pseudostem is a peculiarity of the herbaceous banana plant which consists of closely packed leaf-petiole sheaths (Saravanan and Aradhya, 2011). It provides a unique microhabitat for endophytic microorganisms and was recently identified as a bacterial hot spot colonized by an extraordinary high abundance and diversity of enterics (Rossmann et al., 2012). Consequently, we hypothesized a key role of the Enterobacteriaceae for plant health especially in the endophytic microenvironments. To additionally capture the group of often plant-beneficial fluorescent pseudomonads (Ayyadurai et al., 2006; Weller, 2007), and at the same time to preserve the necessary sequencing depth, we decided to focus on the whole gammaproteobacterial fraction by employing a comprehensive 16S rRNA gene amplicon sequencing approach. Comparisons between colonization patterns reveal the impact of (i) biogeography (Nicaragua vs. Costa Rica), and (ii) agroforestry conditions (banana-coffee intercropping with vs. without agroforestry) on the banana-associated gammaproteobacterial microbiota.

\section{MATERIALS AND METHODS EXPERIMENTAL DESIGN AND SAMPLING PROCEDURE}

Samples were taken in November 2012 from M. acuminata Colla (AAA group) cultivar Gros Michel in Nicaragua and Costa Rica. In each country, samples of banana roots, pseudostem, leaves, and rhizosphere soil were collected from three different farms (Figure S1), where bananas were cultivated in intercropping systems with Coffea spp. To understand the effect of agroforestry on the banana-associated microbiome, samples were collected on each farm from sites with and without associated Fabaceae trees. The predominant trees were Inga spp. in Nicaragua and Erythrina poeppigiana in Costa Rica. Each site was under the respective production system since more than 50 years. Composite samples consisting of sub-samples from five appropriate plants without visible infestation of any disease were taken for each microenvironment.

\section{TOTAL COMMUNITY DNA ISOLATION}

For extraction of metagenomic DNA from the rhizosphere, $2 \mathrm{~g}$ of rhizospheric soil were mixed with $15 \mathrm{ml}$ of $0.85 \% \mathrm{NaCl}$ for
$10 \mathrm{~s}$ on the vortex. To isolate total community DNA from the endorhiza, $5 \mathrm{~g}$ of roots were surface-sterilized with $4 \% \mathrm{NaOCl}$ for $5 \mathrm{~min}$. Afterwards, roots were washed three times with sterile distilled water and transferred to sterile WhirlPaks (Nasco, Fort Atkinson, USA), then $10 \mathrm{ml}$ of $0.85 \% \mathrm{NaCl}$ were added and the surface-sterilized roots were homogenized using mortar and pestle. Pseudostem samples $(5 \mathrm{~g})$ were washed with sterile distilled water, transferred to WhirlPaks, and after $10 \mathrm{ml}$ of $0.85 \% \mathrm{NaCl}$ were added, homogenized with mortar and pestle. From phyllosphere samples, $5 \mathrm{~g}$ of leaves were washed three times with sterile distilled water, before homogenization with $10 \mathrm{ml}$ of $0.85 \% \mathrm{NaCl}$. From the liquid parts $4 \mathrm{ml}$ were centrifuged at high speed $\left(16,000 \times \mathrm{g}, 4^{\circ} \mathrm{C}\right)$ for $20 \mathrm{~min}$ and resulting pellets were stored at $-70^{\circ} \mathrm{C}$. Total community DNA was extracted using the FastDNA SPIN Kit for Soil (MP Biomedicals, Solon, USA) according to the manufacturer's protocol. Metagenomic DNA samples were encoded using abbreviations indicating: (1) country (N-, Nicaragua; $\mathrm{C}-$, Costa Rica), (2) microenvironment (S, rhizosphere soil; Re, endorhiza; Ps, pseudostem; L, leaves), (3) farm (1-3 in each country; Figure S1) (4) agroforestry conditions ( $\mathrm{T}+$, with trees; $\mathrm{T}-$, without trees).

\section{GAMMAPROTEOBACTERIAL 16S rRNA GENE PROFILING BY ILLUMINA MiSeq SEOUENCING}

For a deep-sequencing analysis of the banana-associated Gammaproteobacteria community, the hypervariable V4 region of the 16S rRNA gene was amplified in a nested PCR approach with the Gammaproteobacteria specific primer pair Gamma395f/Gamma871r (Mühling et al., 2008) and the universal primer pair 515F/806R (Caporaso et al., 2011), which carried sample specific tags. The reaction mixture for the first PCR $(20 \mu \mathrm{l})$ contained $1 \times$ Taq\&Go (MP Biomedicals, Eschwege, Germany), $2 \mathrm{mM} \mathrm{MgCl}_{2}, 0.1 \mu \mathrm{M}$ of each primer and $1 \mu \mathrm{l}$ of template DNA dilution $\left(96^{\circ} \mathrm{C}, 4 \mathrm{~min} ; 30\right.$ cycles of $96^{\circ} \mathrm{C}, 1 \mathrm{~min} ; 54^{\circ} \mathrm{C}$, $1 \mathrm{~min} ; 74^{\circ} \mathrm{C}, 1 \mathrm{~min}$; and elongation at $\left.74^{\circ} \mathrm{C}, 10 \mathrm{~min}\right)$. The second PCR $(30 \mu \mathrm{l})$ was performed by using $1 \times$ Taq\&Go, $0.2 \mu \mathrm{M}$ of each primer and $1.2 \mu \mathrm{l}$ from dilutions of the first PCR mixtures $\left(94^{\circ} \mathrm{C}, 3 \mathrm{~min} ; 32\right.$ cycles of $94^{\circ} \mathrm{C}, 45 \mathrm{~s} ; 60^{\circ} \mathrm{C}, 1 \mathrm{~min} ; 72^{\circ} \mathrm{C}$, $18 \mathrm{~s}$; and elongation at $\left.72^{\circ} \mathrm{C}, 10 \mathrm{~min}\right)$. PCR products of three independent reactions were pooled in equal volumes and purified by employing the Wizard SV Gel and PCR Clean-Up System (Promega, Madison, USA). Sequence libraries were generated by a paired-end approach using the Illumina MiSeq platform (Eurofins MWG, Ebersberg, Germany). The nucleotide sequences are available in the European Nucleotide Archive (www.ebi.ac.uk/ ena) under the accession number PRJEB8107.

Data analysis was performed by employing the open source software package QIIME 1.8 (Caporaso et al., 2010a). Sequencing reads with more than three consecutive low quality base calls (Phred quality score $\leq 20$ ) were truncated at the position where their quality began to drop, and only reads with $>75 \%$ consecutive high quality base calls, without any ambiguous characters, and longer than 200 nucleotides in length were retained for further analyses. All quality sequences were adjusted in the same orientation and clustered into operational taxonomic units (OTUs) with uclust (Edgar, 2010), using 3, 5, and 10\% dissimilarity thresholds. From each OTU the most abundant sequence 
was selected as the representative one, and the taxonomy of the representative set was assigned with the uclust-based consensus taxonomy assigner using an $80 \%$ confidence threshold. The representative sequence set was aligned with PyNAST (Caporaso et al., 2010b). Chimera check was performed with ChimeraSlayer and potentially chimeric sequences were discarded. OTU tables at the different dissimilarity levels were constructed, and OTUs not assigned to the class of Gammaproteobacteria as well as singletons were removed from the dataset. For alpha and beta diversity analyses, OTU tables were rarefied at 13,610 reads. Diversity indices Shannon (Shannon, 1997) and Chaol (Chao and Bunge, 2002) were determined based on the normalized clustering data. Significant differences were calculated with PASW Statistics 18 (SPSS Inc., Chicago, IL, USA) using Tukey and Games-Howell post hoc tests, depending on the homogeneity of variances. Beta diversity was analyzed based on weighted UniFrac distances (Lozupone et al., 2007) and 10 jackknife replicates of the total rarefied datasets. Statistical analyses were performed using the adonis test with 999 permutations. Taxonomy based ring-charts were created with Krona 2.2 (Ondov et al., 2011).

Profile clustering network analyses were performed in order to highlight single taxonomic groups corresponding to genus level (OTUs at a dissimilarity level of 3\% summarized at taxonomic level 6) with considerable differences between banana plants grown in Nicaragua and in Costa Rica and between those grown with and without associated trees. The network analyses were carried out with taxa exhibiting a mean read change of more than $0.2 \%$ of the data set. If the ratio of relative mean abundances exceeded 1.5, the taxa were regarded as altered and assigned to the respective profile. Networks depicting community changes resulting from biogeographical location were restricted to taxa which significantly differed between countries. Significant differences were ascertained with Metastats (White et al., 2009), where $p$-values were computed using a combination of the nonparametric $t$-test, exact Fisher's test, and the false discovery rate with $10^{3}$ permutations. For networks showing differences caused by agroforestry, only taxonomic groups featuring the same pattern in all three farms of a country were considered. Visualization of the networks was carried out using Cytoscape 2.8.3 (Smoot et al., 2011).

\section{RESULTS}

\section{RICHNESS AND DIVERSITY OF THE BANANA-ASSOCIATED GAMMAPROTEOBACTERIAL COMMUNITY}

The gammaproteobacterial microbiota associated to the rhizosphere, endorhiza, pseudostem, and foliage of healthy banana plants grown under different agroforestry conditions in Nicaragua and Costa Rica analyzed by a barcoded 16S rRNA gene amplicon sequencing approach based on Illumina MiSeq sequencing yielded in 2,234,043 quality sequences with a read length $\geq 200$ nucleotides, between 13,619 and 111,332 quality reads per sample. Rarefaction analyses of the sequencing libraries at a genetic dissimilarity level of 3\% are depicted in Figure S2. Comparisons of observed OTUs with their estimated richness by the Chaol index revealed coverage between 87.3 and $47.4 \%$ per sample at order level (Table S1). The sequencing efforts at

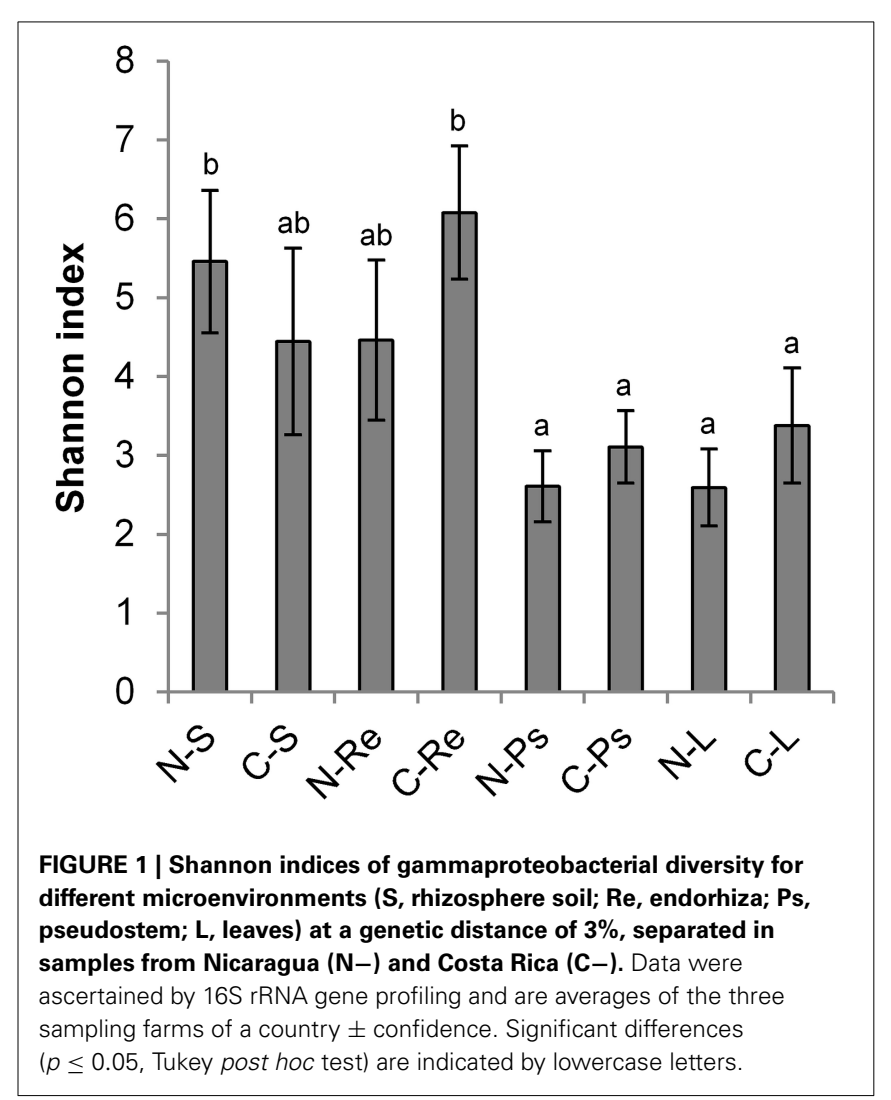

genus and species level reached $74.1-39.9 \%$ and $68.8-31.5 \%$, respectively. The computed Shannon indices of diversity $\left(\mathrm{H}^{\prime}\right)$ ranged from 7.56 to 1.47 at a genetic distance of $3 \%$ (Table S1). In general, rhizosphere and endorhiza samples exhibited higher gammaproteobacterial diversity than pseudostem and leaves (Figure 1). Within samples from Nicaragua, the highest values were observed for the rhizospheric soil (5.46 on average \pm 0.90 confidence), but without a significant difference ( $p \leq 0.05$, Tukey post hoc test) to the endorhiza $(4.46 \pm 1.02)$. Significantly lower Shannon indices than in the rhizosphere soil were detected for pseudostem (2.61 \pm 0.45$)$ and leaves samples $(2.59 \pm 0.49)$. Banana plants from Costa Rica revealed the highest diversity in the endorhiza $(6.08 \pm 0.85)$, which not significantly differed from the rhizosphere soil $(4.45 \pm 1.18)$. Significantly lower values than in the endorhiza were observed for leaves $(3.38 \pm 0.73)$ and pseudostem $(3.11 \pm 0.46)$. Between the same microenvironments of banana plants from the two countries, no significant differences were observed. Agroforestry did not show a significant impact $(p \leq 0.05$, Games-Howell post hoc test) on the gammaproteobacterial diversity of the different microenvironments.

\section{TAXONOMIC COMPOSITION OF THE GAMMAPROTEOBACTERIAL BANANA MICROBIOME}

Nearly all quality sequences could be assigned below the class level, and over all banana-associated communities, high abundances of Pseudomonadales, Enterobacteriales, Xanthomonadales, and Legionellales were found (Figures 2, 3). The rhizosphere 


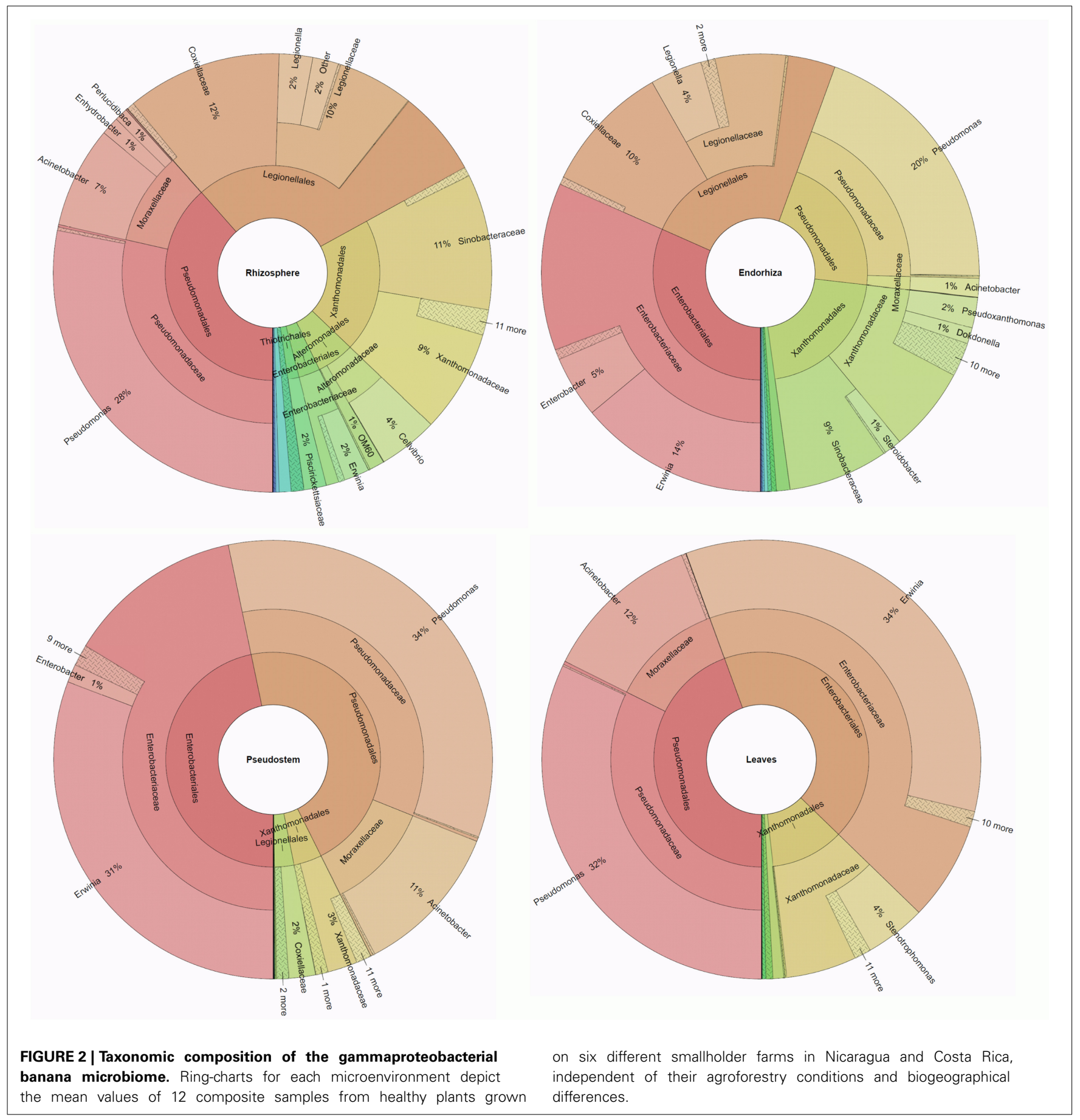

of bananas from Nicaragua was colonized by a significantly higher abundance ( $p \leq 0.05$, Metastats) of Pseudomonadales, Thiotrichales, as well as of unclassified Gammaproteobacteria than the rhizosphere soil of Costa Rica. Conversely, the plant rhizosphere from Costa Rica was inhabited to a greater extent of Legionellales and Enterobacteriales. The endorhiza of bananas from Nicaragua exhibited significantly higher relative abundances of Pseudomonadales, while Xanthomonadales occurred in higher abundances in endorhiza samples from Costa Rica. The

pseudostem in general was highly dominated by Enterobacteriales and Pseudomonadales and showed no significant differences between countries at order level. The foliage exhibited a similar gammaproteobacterial colonization to the pseudostem. However, the leaves from Costa Rica revealed in addition to the dominant orders higher abundances of Oceanospirillales than those from Nicaragua.

At lower taxonomic levels, Pseudomonadales could be assigned to Pseudomonadaceae (genus Pseudomonas) and Moraxellaceae 

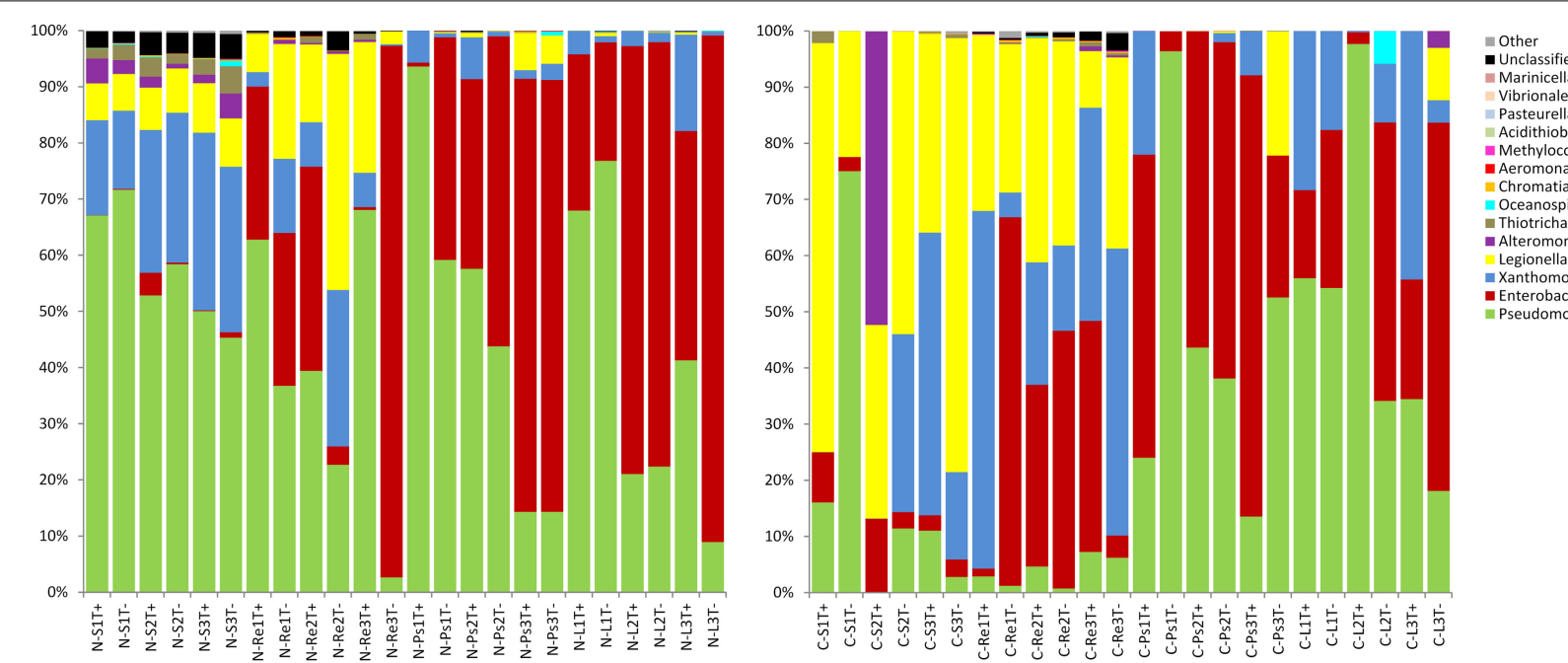

- Unclassified

Vibrionales

Pasteurellales

Methylococcales

- Aeromonadales

Chromatiales

Oceanospirillales
Thiotrichales

Alteromonadales

- Legionellales

- Enterobacteriales

- Pseudomonadales
- Enteriales
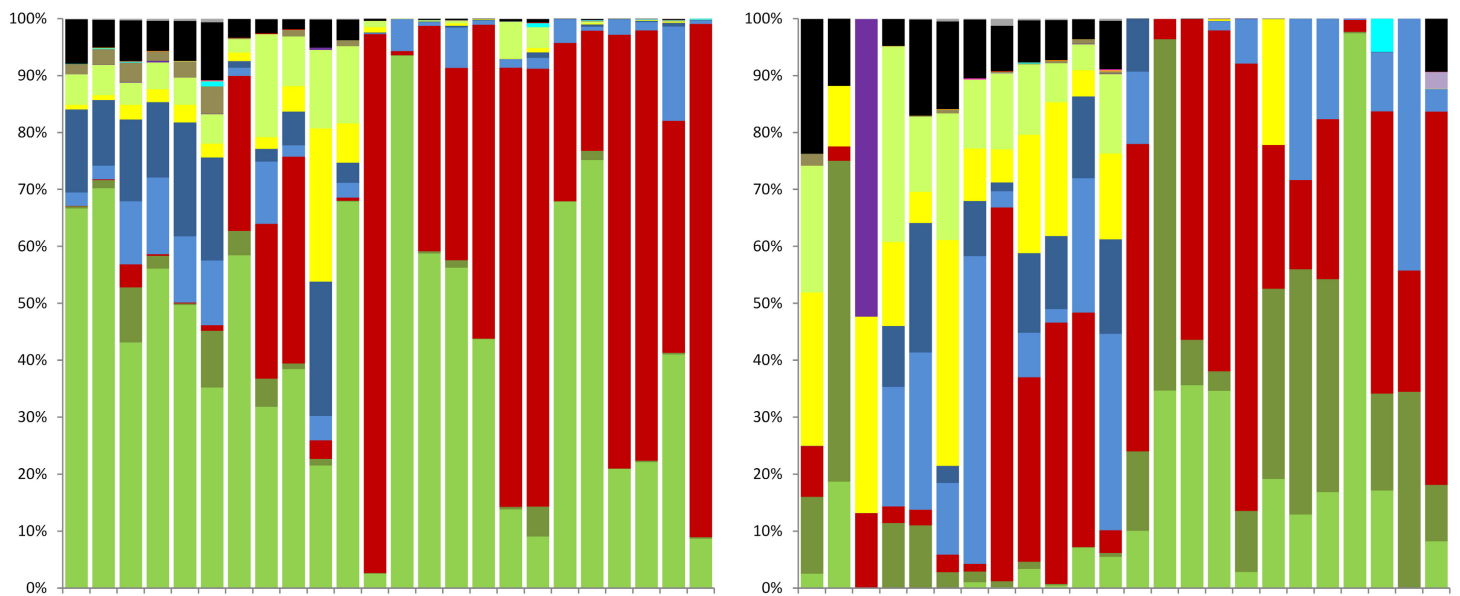

- Other

- Unclassified

Pseudoalteromonadaceae

Vibrionaceae

In Pasteurellaceae

Crenotrichaceae
Methylococcaceae

- Aeromonadaceae

Saccharospirillaceae

Endozoicimonaceae

= Halomonadaceae

Piscirickettsiace
- Colwelliaceae

- Chromatiaceae

Legionellaceae

- Sinobacteracea

Enterobonadacea

- Enterobacteriacea

Pseudomonadacea

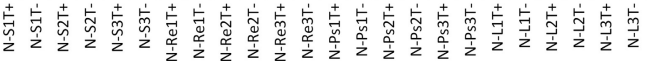
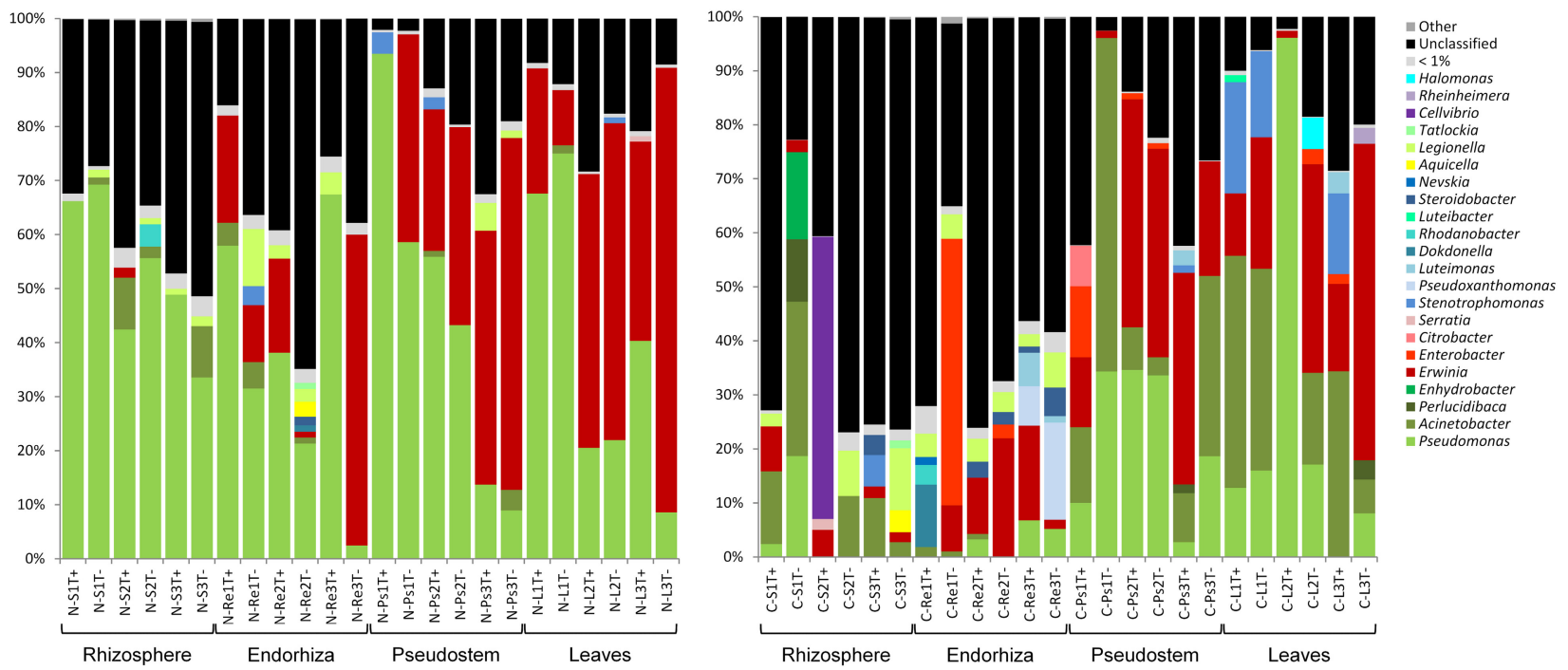

FIGURE 3 | Taxonomic composition of Gammaproteobacteria communities inhabiting rhizosphere, endorhiza, pseudostem, and leaves of banana plants from Nicaragua (left) and Costa Rica (right) grown under different agroforestry conditions. Sequences obtained by Illumina MiSeq sequencing were classified at order, family

and genus level. Samples were encoded using abbreviations indicating (1) country ( $\mathrm{N}-$, Nicaragua; $\mathrm{C}-$, Costa Rica), (2) microenvironment ( $\mathrm{S}$, rhizosphere soil; Re, endorhiza; Ps, pseudostem; L, leaves), (3) farm $(1,2,3)$, and (4) agroforestry conditions ( $T+$, with trees; $T-$, without trees). 
(genera Acinetobacter, Perlucidibaca, and Enhydrobacter), whereby in general Nicaragua samples were highly dominated by Pseudomonadaceae and samples from Costa Rica revealed a high abundance of Moraxellaceae. The enterobacterial fraction was dominated by Erwinia with lower abundances of Enterobacter, Citrobacter, and Serratia. Xanthomonadales sequences could be assigned to different Xanthomonadaceae (Stenotrophomonas, Pseudoxanthomonas, Luteimonas, Dokdonella, Rhodanobacter, and Luteibacter) and Sinobacteraceae (Steroidobacter, and Nevskia). Legionellales could be divided into the families Coxiellaceae (Aquicella) and Legionellaceae (Legionella, and Tatlockia). Further genera identified for taxonomic groups with a relative abundance over $1 \%$ per sample belonged to Alteromonadales (Cellvibrio, and Rheinheimera) and to Oceanospirillales (Halomonas).

\section{IMPACT OF BIOGEOGRAPHY AND AGROFORESTRY}

Considering the total gammaproteobacterial community, no significant differences ( $p \leq 0.05$, adonis test) based on weighted UniFrac distances could be calculated for individual microenvironments between banana plants grown in Nicaragua and Costa Rica (Table S2), and for none of the countries a significant impact on the banana-colonizing Gammaproteobacteria resulting from tree presence was found (Table S3). However, profile clustering network analyses revealed differences of individual taxonomic groups in the colonization patterns between banana plants of the two Central American countries as well as between plants grown in agroforestry systems and those grown without associated trees (Figure 4). Each network subdivides the four investigated microenvironments (rhizosphere soil, endorhiza, pseudostem, and leaves), leaving out taxonomic groups without considerable differences between different conditions. In the networks visualizing the impact of biogeography, only taxa with significant differences ( $p \leq 0.05$, Metastats) between the sampling countries were shown, while in the networks depicting the impact of agroforestry, only taxa featuring the same pattern in all three farms of the respective country were considered.

Without the influence of different agroforestry trees, banana plants from Nicaragua revealed a significantly higher abundance of Pseudomonas, unclassified Sinobacteraceae, Piscirickettsiaceae, and other unclassified Gammaproteobacteria in their rhizosphere (Figure 4A), while the rhizosphere and also the endorhiza from plants in Costa Rica was colonized to a greater extent by Legionellales (unclassified Coxiellaceae and others). The pseudostem did not show significant differences in its gammaproteobacterial colonization between the two countries in plants grown without associated trees. However, the leaves from plants grown in Nicaragua exhibited higher numbers of unclassified Enterobacteriaceae, while those of plants from Costa Rica had higher abundances of Acinetobacter and unclassified Xanthomonadaceae. Under agroforestry conditions, the below-ground habitats of banana plants grown in Nicaragua in association with Inga spp. were characterized by much higher abundances of Pseudomonas than bananas cultivated under agroforestry conditions with E. poeppigiana in Costa Rica (Figure 4B). The rhizosphere of plants grown in the Inga agroforestry system further revealed higher abundances of unclassified Alteromonadales and other unclassified Gammaproteobacteria, while banana plants grown in the Erythrina agroforestry system were more inhabited by unclassified Sinobacteraceae in their endorhiza and by Erwinia in their rhizosphere. Conversely, banana leaves from the Inga agroforestry system in Nicaragua showed a significantly higher number of Erwinia, while the aerial plant parts of Costa Rica's bananas from the Erythrina agroforestry system were colonized to a greater extent by Acinetobacter. In comparison to banana plants grown without associated trees, plants cultivated in agro-ecosystems in Nicaragua harbored an increased number of Pseudomonas (species unclassified) in their endorhiza (Figure 4C), as well as of Xanthomonadaceae (Stenotrophomonas and others) in their above-ground parts. Costa Rica's plants grown in a system without trees revealed a significantly higher number of Erwinia in their phyllosphere than appropriate plants grown in an agroforestry system (Figure 4D).

\section{DISCUSSION}

A deep sequencing analysis of the gammaproteobacterial microbiome associated with the Gros Michel banana variety in Central America revealed an extraordinary high diversity within the endophytic community. Considering the below-ground microhabitats, the endorhiza of plants grown in Nicaragua unveiled a diversity comparable to that of the rhizosphere soil. The succulent pseudostem which can be considered as an above-ground endophytic microhabitat revealed a diversity comparable to that of the leaves encompassing endo- as well as ectophytes. A 16S rRNA gene amplicon sequencing approach targeting only the enterobacterial community of the banana plant revealed a strikingly diverse colonization of its endosphere with a Shannon diversity index for the pseudostem $\left(\mathrm{H}^{\prime}=0.55\right)$ similar to those of rhizosphere samples $\left(\mathrm{H}^{\prime}=0.40-0.55\right)$, even though based on only one pseudostem sample (Rossmann et al., 2012). But normally, what we know from other plants, we face a contrasting picture; due to root exudates and the resulting high nutrient content, the rhizosphere represents a favored microenvironment for microbial colonization and is characterized by a high abundance and diversity (Berendsen et al., 2012; Berg et al., 2014), and only a fraction of this root-associated microorganisms is able to invade, compete with other well-adapted endophytes, and successfully colonize the inner plant tissue (Germaine et al., 2004; Chi et al., 2005). Several endophytes are known for their advantageous associations and close interactions with their host plants. They have been shown to enhance plant growth and quality (Berg et al., 2005a; Köberl et al., 2013), increase plant resistance to abiotic stresses, pathogens and even herbivores (Rodriguez et al., 2009; Marasco et al., 2012; Yi et al., 2013), and contribute to plant-assisted bioremediation (Lodewyckx et al., 2001; Siciliano et al., 2001). The generally high diversity within the endophytic community of the banana plant can be explained by the permanent nature of its corm serving as a reservoir for endophytic diversity and the transmission to following generations via vegetative suckers.

In addition to the diversity, the gammaproteobacterial taxonomic composition was highly similar between the endophytic pseudostem and banana leaves as well, revealing a predominant 
A

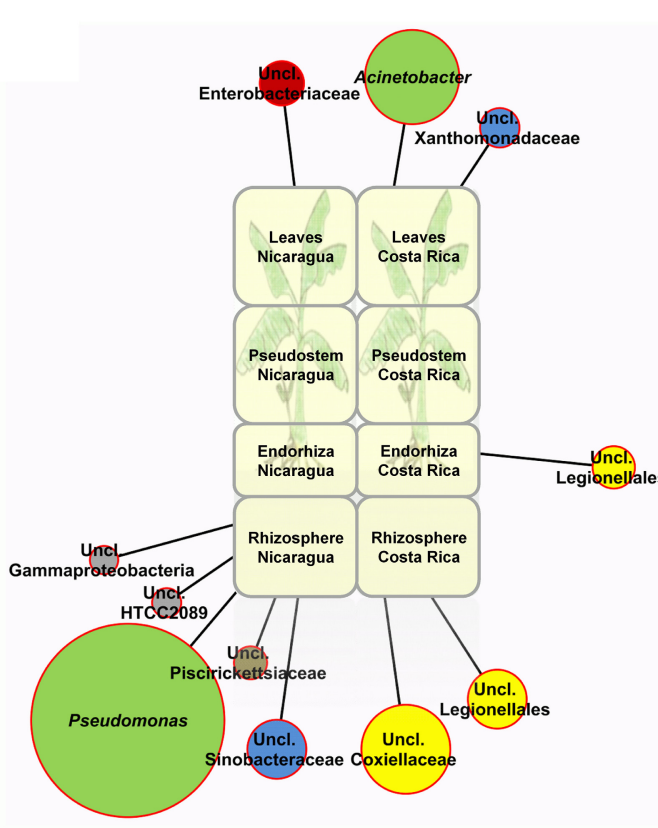

C

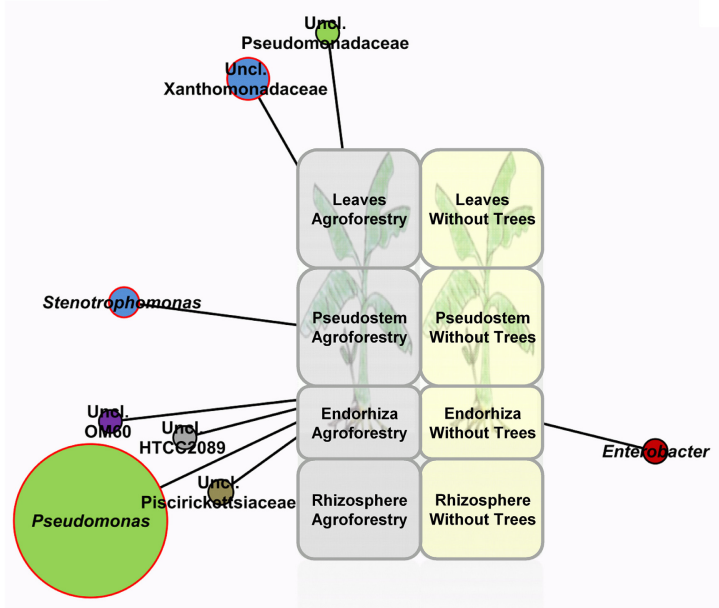

B

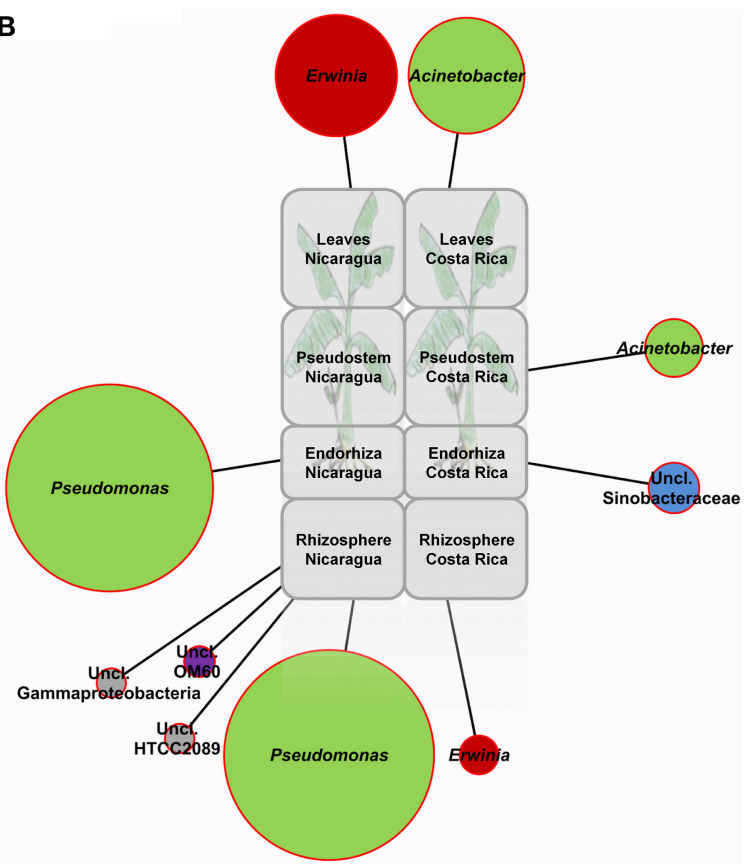

D

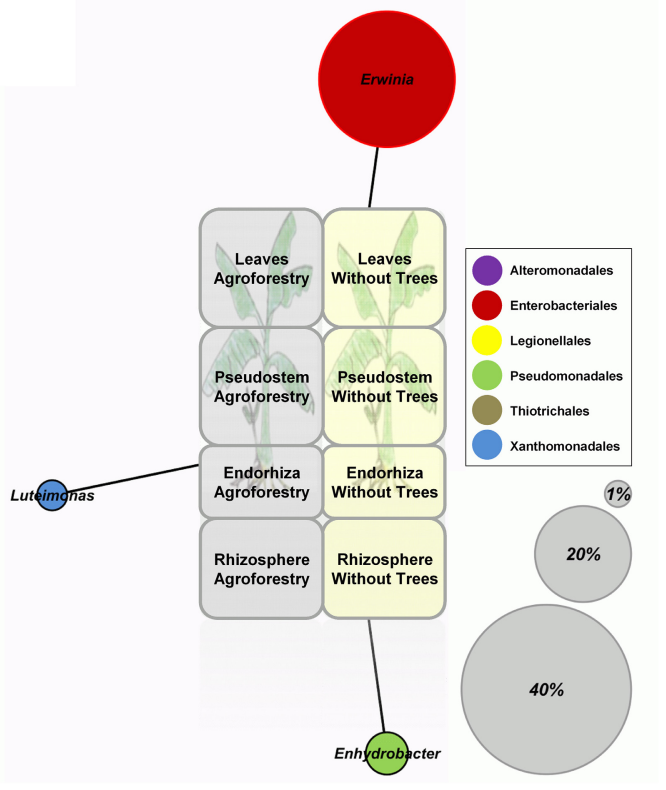

$0.2 \%$ of the data set were used. If the ratio of relative mean abundances exceeded 1.5, the taxa were regarded as altered and assigned to the respective profile. Node sizes correspond to the abundance change between conditions; nodes matching to changes of 1,20 , and $40 \%$ of the data set were added as reference points. $(\mathbf{A}, \mathbf{B})$ Number of depicted nodes was reduced to taxa with significant differences between countries $(p \leq 0.05$, Metastats). (C,D) Only those taxonomic groups that featured the same pattern in all three farms of the respective country are shown. Significant differences are indicated by red node borders. colonization by Enterobacteriaceae and Pseudomonadales. Pseudomonadales, in particular the genus Pseudomonas but also Acinetobacter (both identified as dominant groups in this study), are well-known plant colonizers and among others often accountable for beneficial plant-microbe interactions (Weller,
2007; Rolli et al., 2015). Interestingly, while the Pseudomonadales community in samples from Nicaragua was highly dominated by the genus Pseudomonas, banana plants from Costa Rica revealed significantly higher relative abundances of Acinetobacter. Although it is well-known that the plant microbiome is shaped 
by both soil community and the plant cultivar (Berg and Smalla, 2009), the dominance of enterics in the banana pseudostem described for the East African Highland banana of Uganda (Rossmann et al., 2012) could be confirmed for the Gros Michel variety cultivated in Central America as well and could be extended to the entire perennial above-ground plant parts of the banana. However, while the colonization study of the East African Highland banana in Uganda (Rossmann et al., 2012) revealed Enterobacter as the predominant enterobacterial genus in plant-associated microenvironments, Erwinia was identified as the most dominant genus in the Central American Gros Michel variety. In contrast to Enterobacter which comprises several opportunistic human pathogenic strains (E. aerogenes, E. cloacae) (Berg et al., 2015), Erwinia is mainly known as plant pathogen (E. amylovora, E. tracheiphila) (Eastgate, 2000; Rojas et al., 2013). However, as this study encompassed only healthy banana plants without symptoms of Fusarium wilt or any other disease, there is no indication that the Erwinia strains observed within the banana-associated microbiome are in any manner harmful to the plant. A recent study of the lettuce (Lactuca sativa) microbiome also revealed a preferential occurrence of enterics in the phyllosphere (Erlacher et al., 2014).

In general, a higher impact on the banana-associated gammaproteobacterial microbiome was observed for the biogeographical location than for the agroforestry conditions. The biggest differences between the sampling countries were observed for the rhizosphere communities, representing the most probable source of all other plant colonizers. Consequently, based on the different rhizosphere microbiomes, disparities were found for all investigated microenvironments, whereby above-ground plant parts shared higher similarities, possibly due to a rigorous selection process with subsequent enrichment especially of enterics and pseudomonads. In addition to generally high contents of polyphenols and antioxidants in the succulent banana pseudostem, Saravanan and Aradhya (2011) could recently measure high concentrations of flavonoid compounds. Flavonoids are widely distributed secondary metabolites with diverse metabolic functions in plants; among several others, some of them are wellknown for their antimicrobial activity (Falcone Ferreyra et al., 2012) and have been identified to be involved in the plant-driven selection of microbes (Bais et al., 2006; Weston and Mathesius, 2013).

For both countries and different agroforestry systems, a slight shift of the gammaproteobacterial microbiome resulting from associated Fabaceae trees could be observed. Banana plants grown in the agroforestry system with Inga trees in Nicaragua revealed significantly higher abundances of Pseudomonas and Stenotrophomonas. Both genera comprise several potential plantbeneficial species. For instance, Stenotrophomonas rhizophila has become a model bacterium among the plant growth-promoters and stress protecting agents (Alavi et al., 2013), particularly because of its beneficial effects on plants in salinated soils (Egamberdieva et al., 2011). Positive Pseudomonas-plant interactions are well-known (Weller, 2007) and have already been discussed. However, the genus Pseudomonas also includes some species with potential deleterious effects on plants (P. syringae,
P. viridiflava) (Jakob et al., 2002), and moreover some species of Pseudomonas and also of Stenotrophomonas are known as opportunistic pathogens in humans as well (P. aeruginosa, S. maltophilia). Several studies provided evidence that similar or even identical functions are responsible for the beneficial interactions with plants and virulence in other eukaryotic hosts (Berg et al., 2005b; Alavi et al., 2014). For banana plants grown in association with E. poeppigiana in Costa Rica, a significant decrease of Erwinia spp. was recorded. Although this study targeted exclusively the gammaproteobacterial fraction, results could show that as a consequence of legume-based agroforestry the indigenous banana-associated microbial community was noticeably shifted.

\section{AUTHOR CONTRIBUTIONS}

Conceived and designed the experiments: GB, CS, MD. Performed the experiments: MK, AM. Analyzed the data: MK, MD, GB. Contributed reagents/materials/analysis tools: CS, GB. Wrote the paper: MK, GB.

\section{ACKNOWLEDGMENTS}

This study was supported by the Federal Ministry for Europe, Integration and Foreign Affairs (BMEIA) of the Republic of Austria through the Austrian Development Agency (ADA).

\section{SUPPLEMENTARY MATERIAL}

The Supplementary Material for this article can be found online at: http://www.frontiersin.org/journal/10.3389/fmicb. 2015.00091/abstract

\section{REFERENCES}

Alavi, P., Starcher, M. R., Thallinger, G. G., Zachow, C., Müller, H., and Berg, G. (2014). Stenotrophomonas comparative genomics reveals genes and functions that differentiate beneficial and pathogenic bacteria. BMC Genomics 15:482. doi: 10.1186/1471-2164-15-482

Alavi, P., Starcher, M. R., Zachow, C., Müller, H., and Berg, G. (2013). Rootmicrobe systems: the effect and mode of interaction of stress protecting agent (SPA) Stenotrophomonas rhizophila DSM14405(T.). Front. Plant Sci. 4:141. doi: 10.3389/fpls.2013.00141

Ayyadurai, N., Ravindra Naik, P., Sreehari Rao, M., Sunish Kumar, R., Samrat, S. K., Manohar, M., et al. (2006). Isolation and characterization of a novel banana rhizosphere bacterium as fungal antagonist and microbial adjuvant in micropropagation of banana. J. Appl. Microbiol. 100, 926-937. doi: 10.1111/j.13652672.2006.02863.x

Bais, H. P., Weir, T. L., Perry, L. G., Gilroy, S., and Vivanco, J. M. (2006). The role of root exudates in rhizosphere interactions with plants and other organisms. Annu. Rev. Plant Biol. 57, 233-266. doi: 10.1146/annurev.arplant.57.032905.105159

Berendsen, R. L., Pieterse, C. M., and Bakker, P. A. (2012). The rhizosphere microbiome and plant health. Trends Plant Sci. 17, 478-486. doi: 10.1016/j.tplants.2012.04.001

Berg, G., Eberl, L., and Hartmann, A. (2005b). The rhizosphere as a reservoir for opportunistic human pathogenic bacteria. Environ. Microbiol. 7, 1673-1685. doi: 10.1111/j.1462-2920.2005.00891.x

Berg, G., Erlacher, A., and Grube, M. (2015). “The edible plant microbiome: importance and health issues," in Principles of Plant-Microbe Interactions-Microbes for Sustainable Agriculture, ed B. Lugtenberg (Cham: Springer), 419-426.

Berg, G., Grube, M., Schloter, M., and Smalla, K. (2014). Unraveling the plant microbiome: looking back and future perspectives. Front. Microbiol. 5:148. doi: 10.3389/fmicb.2014.00148

Berg, G., Krechel, A., Ditz, M., Sikora, R. A., Ulrich, A., and Hallmann, J. (2005a). Endophytic and ectophytic potato-associated bacterial communities differ in structure and antagonistic function against plant pathogenic 
fungi. FEMS Microbiol. Ecol. 51, 215-229. doi: 10.1016/j.femsec.2004. 08.006

Berg, G., and Smalla, K. (2009). Plant species and soil type cooperatively shape the structure and function of microbial communities in the rhizosphere. FEMS Microbiol. Ecol. 68, 1-13. doi: 10.1111/j.1574-6941.2009. 00654.x

Butler, D. (2013). Fungus threatens top banana. Nature 504, 195-196. doi: $10.1038 / 504195 a$

Caporaso, J. G., Bittinger, K., Bushman, F. D., DeSantis, T. Z., Andersen, G. L., and Knight, R. (2010b). PyNAST: a flexible tool for aligning sequences to a template alignment. Bioinformatics 26, 266-267. doi: 10.1093/bioinformatics/ btp636

Caporaso, J. G., Kuczynski, J., Stombaugh, J., Bittinger, K., Bushman, F. D., Costello, E. K., et al. (2010a). QIIME allows analysis of high-throughput community sequencing data. Nat. Methods 7, 335-336. doi: 10.1038/ nmeth.f.303

Caporaso, J. G., Lauber, C. L., Walters, W. A., Berg-Lyons, D., Lozupone, C. A., Turnbaugh, P. J., et al. (2011). Global patterns of $16 \mathrm{~S}$ rRNA diversity at a depth of millions of sequences per sample. Proc. Natl. Acad. Sci. U.S.A. 108(Suppl. 1), 4516-4522. doi: 10.1073/pnas.1000080107

Chao, A., and Bunge, J. (2002). Estimating the number of species in a stochastic abundance model. Biometrics 58, 531-539. doi: 10.1111/j.0006341X.2002.00531.x

Chi, F., Shen, S. H., Cheng, H. P., Jing, Y. X., Yanni, Y. G., and Dazzo, F. B. (2005). Ascending migration of endophytic rhizobia, from roots to leaves, inside rice plants and assessment of benefits to rice growth physiology. Appl. Environ. Microbiol. 71, 7271-7278. doi: 10.1128/AEM.71.11.7271-7278.2005

Eastgate, J. A. (2000). Erwinia amylovora: the molecular basis of fireblight disease. Mol. Plant Pathol. 1, 325-329. doi: 10.1046/j.1364-3703.2000. 00044.x

Edgar, R. C. (2010). Search and clustering orders of magnitude faster than BLAST. Bioinformatics 26, 2460-2461. doi: 10.1093/bioinformatics/btq461

Egamberdieva, D., Kucharova, Z., Davranov, K., Berg, G., Makarova, N., Azarova, T., et al. (2011). Bacteria able to control foot and root rot and to promote growth of cucumber in salinated soils. Biol. Fertil. Soils 47, 197-205. doi: 10.1007/s00374-010-0523-3

Erlacher, A., Cardinale, M., Grosch, R., Grube, M., and Berg, G. (2014). The impact of the pathogen Rhizoctonia solani and its beneficial counterpart Bacillus amyloliquefaciens on the indigenous lettuce microbiome. Front. Microbiol. 5:175. doi: 10.3389/fmicb.2014.00175

Falcone Ferreyra, M. L., Rius, S. P., and Casati, P. (2012). Flavonoids: biosynthesis, biological functions, and biotechnological applications. Front. Plant Sci. 3:222. doi: $10.3389 /$ fpls.2012.00222

FAOSTAT (2005). Statistical Database of the Food and Agriculture Organization of the United Nations. Available online at: www.fao.org

Germaine, K., Keogh, E., Garcia-Cabellos, G., Borremans, B., Lelie, D., Barac, T., et al. (2004). Colonisation of poplar trees by $g f p$ expressing bacterial endophytes. FEMS Microbiol. Ecol. 48, 109-118. doi: 10.1016/j.femsec.2003. 12.009

Jakob, K., Goss, E. M., Araki, H., Van, T., Kreitman, M., and Bergelson, J. (2002). Pseudomonas viridiflava and P. syringae - natural pathogens of Arabidopsis thaliana. Mol. Plant Microbe Interact. 15, 1195-1203. doi: 10.1094/MPMI.2002.15.12.1195

Köberl, M., Schmidt, R., Ramadan, E. M., Bauer, R., and Berg, G. (2013). The microbiome of medicinal plants: diversity and importance for plant growth, quality and health. Front. Microbiol. 4:400. doi: 10.3389/fmicb.2013. 00400

Lodewyckx, C., Taghavi, S., Mergeay, M., Vangronsveld, J., Clijsters, H., and van der Lelie, D. (2001). The effect of recombinant heavy metal resistant endophytic bacteria in heavy metal uptake by their host plant. Int. J. Phytoremed. 3, 173-187. doi: 10.1080/15226510108500055

Lozupone, C. A., Hamady, M., Kelley, S. T., and Knight, R. (2007). Quantitative and qualitative beta diversity measures lead to different insights into factors that structure microbial communities. Appl. Environ. Microbiol. 73, 1576-1585. doi: 10.1128/AEM.01996-06

Lundgren, B. (1982). Introduction - what is agroforestry? Agrofor. Syst. 1, 3-12.

Marasco, R., Rolli, E., Ettoumi, B., Vigani, G., Mapelli, F., Borin, S., et al. (2012). A drought resistance-promoting microbiome is selected by root system under desert farming. PLoS ONE 7:e48479. doi: 10.1371/journal.pone. 0048479

Montagnini, F., and Nair, P. K. R. (2004). Carbon sequestration: an underexploited environmental benefit of agroforestry systems. Agrofor. Syst. 61, 281-295. doi: 10.1023/B:AGFO.0000029005.92691.79

Mühling, M., Woolven-Allen, J., Murrell, J. C., and Joint, I. (2008). Improved group-specific PCR primers for denaturing gradient gel electrophoresis analysis of the genetic diversity of complex microbial communities. ISME J. 2, 379-392. doi: 10.1038/ismej.2007.97

Ondov, B. D., Bergman, N. H., and Phillippy, A. M. (2011). Interactive metagenomic visualization in a web browser. BMC Bioinformatics 12:385. doi: 10.1186/1471-2105-12-385

Perrier, X., De Langhe, E., Donohue, M., Lentfer, C., Vrydaghs, L., Bakry, F. et al. (2011). Multidisciplinary perspectives on banana (Musa spp.) domestication. Proc. Natl. Acad. Sci. U.S.A. 108, 11311-11318. doi: 10.1073/pnas.11020 01108

Ploetz, R. C. (2006). Fusarium wilt of banana is caused by several pathogens referred to as Fusarium oxysporum f. sp. cubense. Phytopathology 96, 653-656. doi: 10.1094/PHYTO-96-0653

Rodriguez, R. J., White, J. F. Jr., Arnold, A. E., and Redman, R. S. (2009). Fungal endophytes: diversity and functional roles. New Phytol. 182, 314-330. doi: 10.1111/j.1469-8137.2009.02773.x

Rojas, E. S., Dixon, P. M., Batzer, J. C., and Gleason, M. L. (2013). Genetic and virulence variability among Erwinia tracheiphila strains recovered from different cucurbit hosts. Phytopathology 103, 900-905. doi: 10.1094/PHYTO$11-12-0301-\mathrm{R}$

Rolli, E., Marasco, R., Vigani, G., Ettoumi, B., Mapelli, F., Deangelis, M. L., et al. (2015). Improved plant resistance to drought is promoted by the rootassociated microbiome as a water stress-dependent trait. Environ. Microbiol. doi: 10.1111/1462-2920.12439. [Epub ahead of print].

Rossmann, B., Müller, H., Smalla, K., Mpiira, S., Tumuhairwe, J. B., Staver, C., et al. (2012). Banana-associated microbial communities in Uganda are highly diverse but dominated by Enterobacteriaceae. Appl. Environ. Microbiol. 78, 4933-4941. doi: 10.1128/AEM.00772-12

Saravanan, K., and Aradhya, S. M. (2011). Polyphenols of pseudostem of different banana cultivars and their antioxidant activities. J. Agric. Food Chem. 59, 3613-3623. doi: 10.1021/jf103835z

Seobi, T., Anderson, S. H., Udawatta, R. P., and Gantzer, C. J. (2005). Influence of grass and agroforestry buffer strips on soil hydraulic properties for an Albaqualf. Soil Sci. Soc. Am. J. 69, 893-901. doi: 10.2136/sssaj2004.0280

Shannon, C. E. (1997). The mathematical theory of communication. 1963. MD Comput. 14, 306-317.

Siciliano, S. D., Fortin, N., Mihoc, A., Wisse, G., Labelle, S., Beaumier, D., et al. (2001). Selection of specific endophytic bacterial genotypes by plants in response to soil contamination. Appl. Environ. Microbiol. 67, 2469-2475. doi: 10.1128/AEM.67.6.2469-2475.2001

Smoot, M. E., Ono, K., Ruscheinski, J., Wang, P. L., and Ideker, T. (2011). Cytoscape 2.8: new features for data integration and network visualization. Bioinformatics 27, 431-432. doi: 10.1093/bioinformatics/ btq675

Udawatta, R. P., Kremer, R. J., Garrett, H. E., and Anderson, S. H. (2009). Soil enzyme activities and physical properties in a watershed managed under agroforestry and row-crop systems. Agri. Ecosyst. Environ. 131, 98-104. doi: 10.1016/j.agee.2008.06.001

Weller, D. M. (2007). Pseudomonas biocontrol agents of soilborne pathogens: looking back over 30 years. Phytopathology 97, 250-256. doi: 10.1094/PHYTO97-2-0250

Weston, L. A., and Mathesius, U. (2013). Flavonoids: their structure, biosynthesis and role in the rhizosphere, including allelopathy. J. Chem. Ecol. 39, 283-297. doi: 10.1007/s10886-013-0248-5

White, J. R., Nagarajan, N., and Pop, M. (2009). Statistical methods for detecting differentially abundant features in clinical metagenomic samples. PLoS Comput. Biol. 5:e1000352. doi: 10.1371/journal.pcbi. 1000352

Yi, H. S., Yang, J. W., and Ryu, C. M. (2013). ISR meets SAR outside: additive action of the endophyte Bacillus pumilus INR7 and the chemical inducer, benzothiadiazole, on induced resistance against bacterial spot in field-grown pepper. Front. Plant Sci. 4:122. doi: 10.3389/fpls.2013.00122 
Conflict of Interest Statement: The authors declare that the research was conducted in the absence of any commercial or financial relationships that could be construed as a potential conflict of interest.

Received: 13 November 2014; accepted: 24 January 2015; published online: 11 February 2015.

Citation: Köberl M, Dita M, Martinuz A, Staver C and Berg G (2015) Agroforestry leads to shifts within the gammaproteobacterial microbiome of banana plants cultivated in Central America. Front. Microbiol. 6:91. doi: 10.3389/fmicb.2015.00091
This article was submitted to Plant-Microbe Interaction, a section of the journal Frontiers in Microbiology.

Copyright (C) 2015 Köberl, Dita, Martinuz, Staver and Berg. This is an openaccess article distributed under the terms of the Creative Commons Attribution License (CC BY). The use, distribution or reproduction in other forums is permitted, provided the original author(s) or licensor are credited and that the original publication in this journal is cited, in accordance with accepted academic practice. No use, distribution or reproduction is permitted which does not comply with these terms. 